УДК $620.19+678.5$

02.00.00 Химические науки

ПОВЕРХНОСТНАЯ ЭНЕРГИЯ ПОЛИМЕРОВ
(ЭЛАСТОМЕРНЫХ КОМПОЗИЦИЙ):
СРАВНИТЕЛЬНЫЙ АНАЛИЗ ЗНАЧЕНИЙ
ПОВЕРХНОСТНОЙ ЭНЕРГИИ С
ПАРАМЕТРАМИ ДЕФЕКТНОСТИ
ПОЛИМЕРОВ

Бабаян Александр Львович

Кандидат химических наук

ID автора (РИНЦ спин код: 5810-6890)

Краснодарское высшее военное авиационное

училище летчиков имени Героя Советского Союза

А.К.Серова

350090, Россия, г. Краснодар-5, ул. Дзержинского,

d. 135

e-mail: amb_2004@mail.ru

Поверхностная энергия полимеров определяет такие важные свойства полимеров как смачиваемость, адгезию, способность к сорбции низкомолекулярных веществ. В теоретическом плане она отражает на макроуровне строение и структуру полимера и, в конечном счете, динамику взаимодействия макромолекулярных цепей. В то же время микродефекты (неоднородности) полимеров, являясь составной частью структуры, при деформировании изменяются и должны вносить свой вклад в изменение поверхностной энергии и её составляющих. В статье решена задача выявления изменений параметров поверхностной энергии полимеров при одноосном деформировании. Представлены данные по количественным показателям поверхностных свойств - общей поверхностной энергии и её дисперсионной и полярной составляющих. Расчёты показали, что дисперсная и полярная составляющие у всех образцов полимерных материалов составляют около $98 \%$ от общей и, следовательно, является определяющей. Впервые получены экспериментальные данные, иллюстрирующие изменение поверхностной энергии полимерных материалов, в том числе и эластомерных композиций при их одноосном деформировании. Относительные изменения поверхностной энергии варьируются от $-54,5 \%$ до $125 \%$. При

деформировании полимерных образцов различной структуры и химической природы наблюдается уменьшение общей поверхностной энергии и её дисперсионной составляющей

Ключевые слова: ПОВЕРХНОСТНАЯ ЭНЕРГИЯ, МИКРОДЕФЕКТНОСТЬ, ДИСПЕРСИОННАЯ И ПОЛЯРНАЯ СОСТАВЛЯЮЩАЯ, ОДНООСНОЕ ДЕФОРМИРОВАНИЕ, ЛОКАЛЬНЫЕ ДЕФОРМАЦИИ
UDC $620.19+678.5$

Chemical sciences

\section{SURFACE ENERGY OF POLYMERS (ELASTOMER COMPOSITIONS): A COMPARATIVE ANALYSIS OF THE VALUES OF SURFACE ENERGY PARAMETERS OF DEFECTS IN POLYMERS}

\author{
Babayan Alexander Lvovich \\ Candidate of Chemistry \\ Author's ID (Spin-code: 5810-6890) \\ Krasnodar Air Force Institute for Pilots named after \\ Hero of the Soviet Union A. K. Serov \\ 350090, Russia, Krasnodar-5, Dzerzhinsky St., 135 \\ e-mail: amb_2004@mail.ru
}

Surface energy of polymers determines such important properties of polymers like wetability, adhesion, ability adsorption of low molecular weight substances. Inner organization, the structure of the polymer and the dynamics of interaction between macromolecular chains are shown at last. At the same time, the micro-defects (inhomogeneity) of polymers, which is an integral part of the structure are changed during deformation and must contribute to changing of the surface energy and its components.

This article solves the task of detecting changes in the parameters of surface energy of polymers under uniaxial deformation. Data for the quantitative indicators of the surface properties total surface energy and its dispersive and polar components are presented. The calculations showed that the disperse and polar components of all the samples of polymer materials are about $98 \%$ of the total and, therefore, is crucial. For the first time experimental data, illustrating the change of surface energy of polymeric materials had been obtained, including elastomeric compositions, when uniaxial deformation. The relative changes of the surface energy vary from 54.5 per cent to $125 \%$. A decrease of total surface energy and its dispersion component is observed during deformation of polymer samples of different structure and chemical nature

Keywords: SURFACE ENERGY, MICRO

DEFECTS, DISPERSIVE AND POLAR

COMPONENT, UNIAXIAL DEFORMATION, LOCAL DEFORMATION 
Doi: 10.21515/1990-4665-131-029

\section{Поверхностная энергия полимеров (эластомерных композиций):}

\section{сравнительный анализ значений поверхностной энергии с параметрами дефектности полимеров}

Поверхностная энергия полимеров определяет такие важные свойства полимеров как смачиваемость, адгезию, способность к сорбции низкомолекулярных веществ. В теоретическом плане отражает на макроуровне строение и структуру полимера и, в конечном счете, динамику взаимодействия макромолекулярных цепей. В то же время микродефекты (неоднородности) полимеров, являясь составной частью структуры, при деформировании изменяются и должны вносить свой вклад в изменение поверхностной энергии и её составляющих, поэтому, основными структурными элементами нашей работы явились: определение параметров поверхностной энергии исходных образцов; изменение параметров поверхностной энергии при деформировании; взаимосвязь значений поверхностной энергии с параметрами дефектности полимеров.

Как известно /1,2/, наиболее близкие по значению данные о поверхностной энергии получены по равновесным краевым углам смачивания, поэтому этот метод и был применён в данной работе. На основе этого способа нами была разработана методика растяжения полимерных образцов и определения краевых углов смачивания.

Исследования поверхностных явлений в полимерах заключалось в следующем.

Вырезались образцы длиной рабочего участка 50 мм и шириной 10 мм и подвергались обезжириванию, промывке водопроводной водой с мылом, обработка этиловым спиртом и сушке. 
Определение краевых углов смачивания заключалось в следующем. Образец с рабочими размерами (50х10 мм) горизонтально крепится на устройстве для одноосного деформирования образцов в зажимах. Далее, после нанесения капли, переноса ее контура на бумагу и определения радиуса и высоту капли, переходим к удлинению образца.

Образец удлиняется на определённую величину с помощью возвратно-потупательного устройства и выдерживается 15 мин в деформированном состоянии, затем повторяются действия, изложенные выше.

Время выдержки образца в деформированном состоянии определяется экспериментально, и заключается в следующем: на деформированный образец наносится капля одной и той же жидкости и определяется её краевые углы смачивания с интервалом в 1 минуту до тех пор, пока не станет ясно, что размеры капли перестали изменяться, этот момент и станет временем выдержки образца.

Затем по формулам (1) и (2) определяются краевые углы смачивания

$$
\begin{gathered}
\operatorname{tg} \theta=(2 r H) /\left(r^{2}-H^{2}\right), \text { при }\left(\theta<90^{\circ}\right) \\
\operatorname{tg} \theta=\sqrt{2 r H-H^{2}} /(r-H), \text { при }\left(\theta>90^{\circ}\right),
\end{gathered}
$$

где $\mathrm{r}$ - радиус капли, мм; Н - высота капли, мм.

Для получения более точных результатов по определению краевого угла смачивания делается по 4 - 5 параллельных опыта. После чего наиболее отличные результаты отбрасываются, а остальные усредняются.

Поверхностную энергию принято считать величиной, состоящей из двух компонентов - дисперсионной и полярной (последняя включает в себя силы недисперсионной природы), поэтому по известному уравнению Оуэнса и Вендта, где используется краевой угол смачивания, были определены эти составляющие и соответственно суммарная поверхностная энергия (формула 3): 


$$
1+\cos \theta=\frac{2\left(\gamma_{m}^{Д}\right)^{1 / 2}\left(\gamma_{\varkappa}^{\text {म }}\right)^{1 / 2}}{\gamma_{\varkappa}}+\frac{2\left(\gamma_{m}^{P}\right)^{1 / 2}\left(\gamma_{\varkappa}^{P}\right)^{1 / 2}}{\gamma_{\varkappa}},
$$

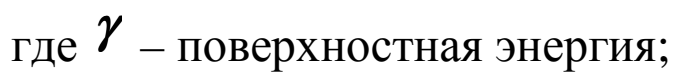

$\theta \quad$ - краевой угол смачивания;

$\gamma_{i}^{j}$ - дисперсионная (Д) и полярная (P) составляющие поверхностной энергии, характеризующие твёрдое тело (т) либо жидкость (ж).

Путём несложных алгебраических преобразований это уравнение приводится к виду $\boldsymbol{y}=\boldsymbol{a x}+\boldsymbol{b}$, где:

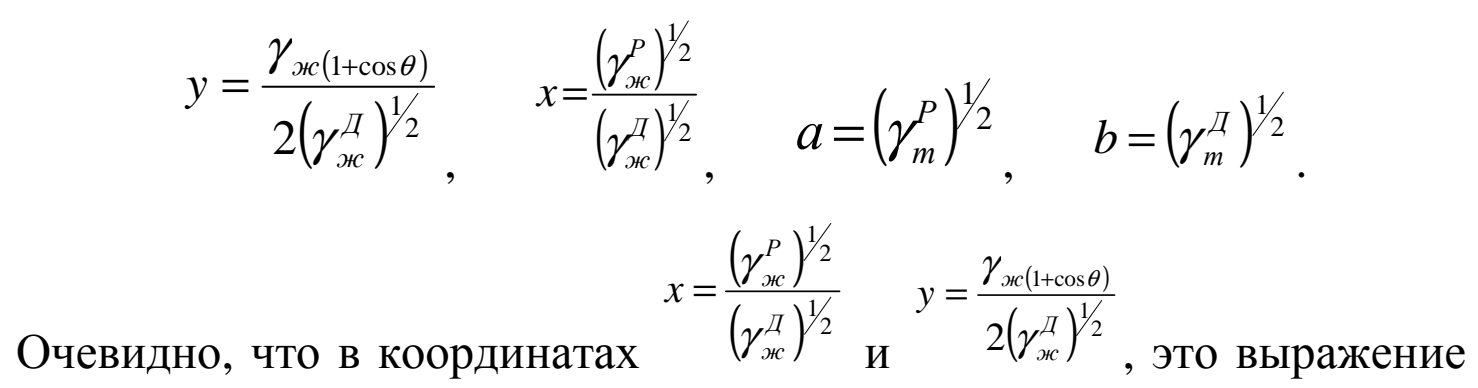
может быть представлено, как прямая tg угла наклона, который есть корень квадратный из полярной составляющей поверхностной энергии твёрдого тела:

$$
\operatorname{tg} \theta=\left(\gamma_{m}^{P}\right)^{1 / 2}
$$

а отсекаемый по оси ординат отрезок, есть корень квадратный из дисперсионной составляющей поверхностной энергии твёрдого тела $\left(\gamma_{m}^{Д}\right)^{1 / 2}$

Общая поверхностная энергия твёрдого тела определяется суммой этих составляющих:

$$
\gamma_{m}=\gamma_{m}^{Д}+\gamma_{m}^{P}
$$

Были проведены эксперименты по оценке параметров поверхностной энергии полимерных материалов. 
В таблице 1 представлены экспериментальные и литературные /3/ значения поверхностной энергии и её составляющих некоторых полимерных материалов.

Таблица 1 - Поверхностная энергия полимерных материалов

\begin{tabular}{|c|c|c|c|c|}
\hline Полимер & $\begin{array}{c}\gamma^{\mathrm{d}}, \\
\mathrm{m} \text { Дж/м }\end{array}$ & 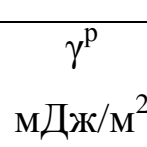 & 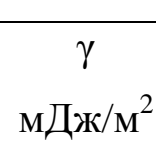 & $\begin{array}{c}\gamma^{*} \\
\text { мДж/ } / \mathrm{M}^{2}\end{array}$ \\
\hline ПЭНП & 30 & 0,1 & 30,1 & 31 \\
\hline$\Pi Э Н \Pi_{\text {тл. разр. }}$ & 15 & 27 & 42 & \\
\hline$\Pi \mathrm{A}-12$ & 39 & 0,04 & 39,04 & \\
\hline$\Phi-4$ & 17 & 0,2 & 17,2 & 19 \\
\hline ПВХ & 38 & 1,2 & 39,2 & 41,5 \\
\hline ПС & 32 & 0,1 & 32 & 31 \\
\hline ПИ & 24 & 0,1 & 24,1 & \\
\hline ПЭТФ & 45 & 0,2 & 45,2 & 47,3 \\
\hline
\end{tabular}

$\gamma^{*}$ - литературные данные.

Анализ таблицы 1 позволяет сделать вывод о том, что полученные нами значения общей поверхностной энергии достаточно близки к литературным значениям.

Рассматривая дисперсную и полярную составляющие общей поверхностной энергии, необходимо отметить, что у всех образцов полимерных материалов дисперсная составляющая составляет около $98 \%$ от общей и, следовательно, является определяющей.

Подобный же вывод можно сделать, проанализировав таблицу 2, где представлены значения поверхностной энергии и её составляющих резин.

Таблица 2 - Поверхностная энергия эластомерных композиций

\begin{tabular}{|c|c|c|c|}
\hline Эластомер &  & $\begin{array}{c}\gamma^{\mathrm{p}} \\
\mathrm{M} Д ж / \mathrm{M}^{2}\end{array}$ & $\begin{array}{c}\gamma \\
\mathrm{м} Д ж / \mathrm{m}^{2}\end{array}$ \\
\hline HK & 34 & 0,06 & 34,06 \\
\hline Б -201 & 33 & 0,02 & 32,02 \\
\hline PC-26 & 23 & 19 & 42 \\
\hline PC-26 фторир.. & 6 & 32 & 38 \\
\hline БК & 19 & 0,2 & 19,2 \\
\hline
\end{tabular}


В данной работе также приведены значения общей поверхностной энергии и её составляющих для ПЭНП, обработанного в тлеющем разряде и модифицированного эластомера - фторированный образец резины РС-26 на основе СКН-26.

Так значение общей поверхностной энергии ПЭНП обработанного в тлеющем разряде увеличилось по сравнению с исходным образцом, а у образца резины РС-26 уменьшилось. Анализ литературных и экспериментальных данных позволяют сделать вывод о том, что используемый нами метод является весьма информативным и достоверным.

В литературе отсутствуют экспериментальные данные об изменении поверхностной энергии при деформации полимеров. Нами проведены экспериментальные исследования изменения параметров поверхностной энергии образцов различных полимеров при одноосной деформации.

Анализ полученных данных позволяет отметить следующее. При деформировании полимерных образцов различной структуры и химической природы наблюдается уменьшение или прослеживается тенденция к уменьшению общей поверхностной энергии и её дисперсионной составляющей, т. е. определяющим является изменение дисперсионной составляющей поверхностной энергии.

Изменение полярной составляющей, хотя и не определяет общее изменение поверхностной энергии, носит неоднозначный характер для разных полимеров. Так, для ПА-12, ПЭНП, ПЭТФ ( $\delta=6$ мкм) параметр $\gamma^{\mathrm{p}}$ возрастает; для ФП-4 и ПВХ уменьшается, для ПС, ПИ и ПЭТФ $(\delta=100$ и 350 мкм) сначала происходит уменьшение, а затем возрастание $\gamma^{\mathrm{p}}$.

Для объяснения полученных результатов, необходимо учитывать некоторые обстоятельства. Теоретические представления о поверхностной энергии твёрдых полимеров весьма скудны и не опираются на достаточные термодинамические или структурные основания. Учёт специфики 
строения полимерных тел и макромолекул пока отсутствует. Можно указать, по крайней мере, на два обстоятельства, которые будут определять поверхностные свойства полимерных тел. Это гибкость цепи и полидисперсность.

Первый фактор должен приводить к изменению конфигурационного набора макромолекул в поверхностном слое, а отсюда к изменению его плотности и в том числе к возможности изменения поверхностных свойств при различных часто физических взаимодействиях, в том числе и механических. Полидисперсность полимеров при одинаковой средней молекулярной массе также будут вносить существенный вклад в величину поверхностной энергии вследствие различий в поверхностной активности и поверхностной энергии отдельных фракций.

Известно, что существует связь между плотностью энергии когезии (ПЭК) и поверхностной энергией, т. е. в конечном счете, между плотностью и поверхностной энергией. Но эта взаимосвязь не является линейной. Кроме того, необходимо иметь ввиду, что объёмная плотность полимера и плотность поверхностных слоёв (поверхностная плотность) не являются одинаковыми величинами. Исходя из вышесказанного, можно утверждать, что при одноосном деформировании полимеров поверхностная плотность образцов полимеров будет уменьшаться, что и определяет уменьшение дисперсионной составляющей и общей поверхностной энергии. Изменение полярной составляющей поверхностной энергии определяется совокупностью процессов изменения поверхностной плотности, наличием полярных групп в полимерах и возможностью появления радикалов при деформации полимеров.

На разных стадиях деформирования могут преобладать те или другие из рассмотренных процессов, что и определяет неоднозначное изменение параметра $\gamma^{\mathrm{p}}$ для различных полимеров. 
Изучение изменения параметров поверхностной энергии полимеров при одноосном деформировании являлось, с одной стороны, попыткой установить новые экспериментальные факты, а, с другой стороны, попытаться создать некоторую базу для осмысления и понимания изменения поверхностной энергии при деформировании каучуков и резин на их основе.

При проведении сравнительного анализа изменения параметров поверхностной энергии с изменением параметров дефектности полимеров в сопоставимых условиях, мы руководствовались следующими соображениями.

Во-первых, параметры микродефектности это свойство объёма (матрицы) полимера, а параметры свободной энергии определяются поверхностными свойствами полимеров. Во-вторых, параметры микродефектности, характеризующие микродефектность, должны тем или иным способом влиять на поверхностные свойства, такие как поверхностная плотность материалов, возможность изменения истинных значений геометрии поверхности образцов и т.д.

Таким образом, мы попытались провести такой анализ для исходных образцов; образцов, подвергнутых подвергших одноосному растяжению (воздействию механических полей).

Анализируя результаты сравнительного анализа для исходных образцов материалов, необходимо признать, что сравнительный анализ по параметрам поверхностной энергии является не совсем корректным, поскольку образцы материалов различаются по химическому составу, структуре и строению, различным химическим добавкам. В защиту такого анализа следует привести тот факт, что определяющей поверхностную энергию всех исследованных материалов является дисперсионная составляющая, а дисперсионные силы в полимерах в основе носят общий характер. 
Можно утверждать о некоторой общей тенденции, а именно, что с увеличением плотности микродефектов (или предельного адсорбционного объёма) общая поверхностная и её дисперсионная составляющая возрастают, причём, в первом приближении, этот эффект начинает проявляться при увеличении параметра W выше $2 \times 10^{-3} \mathrm{~cm}^{3} / \Gamma / 4 /$.

При анализе результатов сравнительного анализа зависимости значения полярной составляющей от плотности дефектов, наблюдается обратная картина: с увеличением плотности дефектов полярная составляющая имеет слабо выраженную склонность к уменьшению.

При анализе результатов экспериментальных данных по зависимости параметров $\gamma$ и $\gamma^{\mathrm{d}}$ от среднего условного размера микродефекта $\mathrm{k}$, можно сделать вывод о том, что с увеличением параметра $\mathrm{k}$ параметры $\gamma$ и $\gamma^{\mathrm{d}}$ имеют тенденцию к уменьшению. Можно предположить следующее объяснение полученным результатам.

Если выделить для анализа единицу поверхности образца полимерного материала, то можно предположить, что с общим увеличением микродефектности, суммарная площадь микродефектов, находящихся на поверхности также будут возрастать, тогда по сравнению с идеальной (бездефектной) поверхностью истинная площадь образца будет тем больше, чем больше микродефектность, а это должно вести к увеличению плотности нескомпенсированных дисперсионных сил и, следовательно, к увеличению дисперсионной составляющей и общей поверхностной энергии.

В качестве выводов можно утверждать следующее. Для ряда полимерных материалах, в том числе и эластомерных композиций, получены экспериментальные данные по количественным показателям поверхностных свойств - общей поверхностной энергии и её дисперсионной и полярной составляющих. Расчёты показали, что дисперсная и полярная составляющие у всех образцов полимерных 
материалов составляет около $98 \%$ от общей и, следовательно, является определяющей.

Впервые получены экспериментальные данные, иллюстрирующие изменение поверхностной энергии полимерных материалов, в том числе и эластомерных композиций при их одноосном деформировании. Относительные изменения поверхностной энергии варьируются от - 54,5 \% до $125 \%$. При деформировании полимерных образцов различной структуры и химической природы наблюдается уменьшение или прослеживается тенденция к уменьшению общей поверхностной энергии и её дисперсионной составляющей, т. е. определяющим является изменение дисперсионной составляющей поверхностной энергии.

\section{Список литературы}

1. Lyklema, J. The Properties of the Stagnant Layer Unraveled / J. Lyklema, S. Rovillard, and J. De Coninck // Langmuir. - 1998. - Vol.14, №20. - P.5659-5663.

2. Сумм, Б.Д. Гистерезис смачивания / Б.Д. Сумм // Соросовский образовательный журнал. - 1999. - №7. - С.98-102

3. Берлин, А.А. Основы адгезии полимеров / А. А. Берлин, В. Е. Басин. - М.: Химия, 1984. - 319 с.

4. Экспресс-метод оценки параметров пористой структуры адсорбентов и микродефектности полимерных материалов сорбционно-весовым методом. / А.Л. Бабаян, А.Р. Агаев, А.В. Иванов, А.Н. Громов // Тезисы докладов VI Всероссийского симпозиума с участием иностранных ученых. "Актуальные проблемы теории адсорбции и синтеза сорбентов.”-М.: ИФХ РАН, 2000. - 161 с.

\section{References}

1. Lyklema, J. The Properties of the Stagnant Layer Unraveled / J. Lyklema, S. Rovillard, and J. De Coninck // Langmuir. - 1998. - Vol.14, №20. - P.5659-5663.

2. Summ, B.D. Gisterezis smachivanija / B.D. Summ // Sorosovskij obrazovatel'nyj zhurnal. - 1999. - №7. - S.98-102

3. Berlin, A.A. Osnovy adgezii polimerov / A. A. Berlin, V. E. Basin. - M.: Himija, 1984. $-319 \mathrm{~s}$.

4. Jekspress-metod ocenki parametrov poristoj struktury adsorbentov i mikrodefektnosti polimernyh materialov sorbcionno-vesovym metodom. / A.L. Babajan, A.R. Agaev, A.V. Ivanov, A.N. Gromov // Tezisy dokladov VI Vserossijskogo simpoziuma s uchastiem inostrannyh uchenyh. "Aktual'nye problemy teorii adsorbcii i sinteza sorbentov."M.: IFH RAN, 2000. - $161 \mathrm{~s}$. 